\title{
Unconventional association between risk factors and knowledge of stroke among cerebrovascular disease survivors in China: A cross-sectional community-based study (FAST-RIGHT)
}

\section{Shengde Li}

Department of Neurology, Peking Union Medical College Hospital, Peking Union Medical College and Chinese Academy of Medical Sciences

\section{Li-Ying Cui}

Department $\mathrm{f}$ Neurology, Peking Union Medical College Hospital, Chinese Academy of Medical Sciences and Peking Union Medical College

\section{Craig Anderson}

Neurological and Mental Health Division, The George Institute for Global Health, Faculty of Medicine, University of New South Wales, Sydney, Australia. The George Institute for Global Health, Peking University Health Science Center, Beijing, China

\section{Chunpeng Gao}

Disease Control and Prevention Office, Dalian Municipal Central Hospital, Dalian, China

\section{Chengdong Yu}

Department of Epidemiology and Statistics, Institute of Basic Medical Sciences, Chinses Academy of Medical Sciences, Beijing, China

\section{Guangliang Shan}

Department of Epidemiology and Statistics, Institute of Basic Medical Sciences, Chinses Academy of Medical Scicences

\section{Longde Wang}

Stroke Control Project Committee, The National Health Commission, Beijing,China

\section{Bin Peng ( $\sim$ pengbin3@hotmail.com )}

Department of Neurology, Peking Union Medical College Hospital, Peking Union Medical College and Chinese Academy of Medical Sciences, Beijing, China https://orcid.org/0000-0002-4168-3405

\section{Research article}

Keywords: health knowledge, attitudes, practice, awareness, emergency medical services, cerebrovascular disease, survivors, risk factors

Posted Date: June 21st, 2019 
DOI: https://doi.org/10.21203/rs.2.10496/v1

License: (c) (1) This work is licensed under a Creative Commons Attribution 4.0 International License. Read Full License 


\section{Abstract}

Background: Cerebrovascular disease (CVD) survivors are at a risk of recurrent strokes, and early correct response to stroke is crucial to promote access to effective reperfusion therapy. This study aimed to investigate whether there is an association between increased risk factors and the intent to call emergency medical services (EMS) among CVD survivors. Methods: A cross-sectional community-based study was conducted from January 2017 to May 2017, including 187723 adults (age $\geq 40$ years) across 69 administrative areas in China. A CVD survivor population of 6290 was analyzed. Multivariable logistic regression models were used to identify the association between the number of modifiable risk factors and stroke recognition and EMS calling, respectively. Results: The estimated stroke recognition rate in CVD survivors with 0, 1-2, and 3-7 modifiable risk factors was 84.7\% (321/379), 84.4\% (2346/2780), and $85.6 \%(2673 / 3123)$, respectively. The rate of calling EMS in the $0,1-2$, and 3-7 modifiable risk factor groups was $66.0 \%$ (250/379), 62.7\% (1744/2780), and 67.8\% (2117/3123), respectively. The prevalence of cardiovascular diseases was higher among CVD survivors than the non-CVD population. The CVD survivors' knowledge of recognizing stroke and intent to call EMS did not improve with an increasing number of modifiable risk factors, even after adjustment for multiple sociodemographic factors. Conclusions: Despite being at a higher risk of recurrent stroke, Chinese CVD survivors showed poor knowledge of stroke, and their intent to call EMS did not improve with stroke risk. Special, targeted, and enhanced secondary stroke prevention education is needed for CVD survivors.

\section{Introduction}

In China, as in many countries, stroke is a major health challenge, with an increasing number of strokerelated deaths and disabilities [1-4]. Reperfusion therapy with intravenous alteplase, when administered to patients soon after symptom onset, can improve the outcomes of acute ischemic stroke (AIS). However, in clinical settings, less than $2 \%$ of AIS patients receive reperfusion therapy with alteplase in China, mainly due to pre-hospital delays from poor knowledge of stroke $[5,6]$. Emergency medical services (EMS) usage accelerates presentation to the hospital, but a very low proportion of AIS patients use EMS in China [6-9]. Survivors of CVD are prone to a recurrent stroke, which is more severe and disabling [10-12]. However, in our previous report on FAST-RIGHT, a substantial number of CVD survivors (34.6\%) did not call EMS [13]. and the details on stroke recognition and responses among CVD survivors remain unclear. In China, common vascular risk factors such as hypertension, diabetes and dyslipidemia are not well adequately managed [14-16], and many of them probably do not know that their risk factors relate to stroke, which depends on stroke education [17]. The intent to calling EMS seems more important to patients with higher stroke risk. Therefore, based on the FAST-RIGHT study, we aimed to determine the characteristics of CVD survivors, and whether the intent to call EMS increased with the number of modifiable risk factors. We also examined other potential confounders associated with stroke recognition and calling EMS.

\section{Methods}


Data were obtained from the FAST-RIGHT study, which is part of the China National Stroke Screening Survey (CNSSS), and included 69 administrative areas between January 2017 and May 2017. More details on the CNSSS are outlined elsewhere [13, 18], and can also be found on the website of the National Health Commission [19]. The CNSSS was a cross-sectional community-based survey with a 2stage stratified sampling framework based on county-level demographic data. In the FAST-RIGHT study, residents aged 40 years and older were screened by trained research staff using a standard face-to-face questionnaire that covered information about sociodemographic, medical, and family history, lifestyle factors, and four specific questions regarding stroke awareness (See Supplementary Appendix 2, Additional File 1). Commencement of provision of stroke education was also recommended after completing the questionnaire survey. All screening data were transferred from questionnaires to an electronic database and checked centrally for completeness and errors by an experienced data manager. The FAST-RIGHT study was approved by the central ethics committee of Peking Union Medical College Hospital (the principal study center), and all participants provided written informed consent.

\section{Explanatory and Outcome Variables}

Recognition of stroke symptoms was defined as a participant's unprovoked awareness of "facial droop," "arm weakness," and "speech disturbances" (slurred speech, or word-finding difficulties) [20]. Calling EMS immediately after the onset of any of these symptoms was regarded as the correct action in response to a stroke. A reported history of stroke was confirmed by a neurologist or physician, who applied standard diagnostic criteria with any available brain neuroimaging data. The modifiable risk factors included hypertension, diabetes, dyslipidemia, overweight and obesity (BMI=24-50), atrial fibrillation (AF)/valvular heart disease, smoking (including current, former, and passive smoking), and limited exercise (See Supplementary Appendix 3, Additional File 1). The whole group was divided into two subgroups: CVD survivors and non-CVD population. CVD survivors were defined as those with a past history of cerebrovascular disease, including ischemic stroke, cerebral hemorrhage, subarachnoid hemorrhage, and transient ischemic attacks. Individuals without a history of cerebral vascular disease were classified as the non-CVD group.

\section{Statistical Analyses}

The stroke recognition rate (SRR) and correct action rate (CAR) were determined and presented as rates with $95 \%$ confidence intervals $(\mathrm{Cl})$ on the basis of specific subgroups defined by age, sex, site, region, education level, and annual income among CVD survivors. The association between the increased number of modifiable risk factors and stroke recognition, as well as that between the increased number of risk factors and calling EMS were analyzed using multivariable logistic regression. Comparisons of sociodemographic and cardiovascular factors between the CVD survivor and non-CVD groups were performed within the whole group. A standard two-sided $P$ value $(<0.05)$ was considered statistically significant. All analyses were performed using SAS version 9.3. 


\section{Results}

Of the 187,723 residents screened for eligibility, 6,290 were CVD survivors and were included in our analysis. CVD survivors with more risk factors had a higher CAR, but had a similar SRR to those having a single risk factor. The estimated SRR and CAR varied across regions and socioeconomic statuses (Table 1). Across all the different subgroups, CAR was $5.3 \%-29.3 \%$ lower than SRR. Among those that recognized the onset of a stroke, only $67.9 \%$ intended to call EMS (See Supplementary Table S2, Additional File 1).

[Table 1]

Compared to the non-CVD population, CVD survivors made up significantly larger proportions of the elderly and obese patients, as well as participants with lower socioeconomic status (See Supplementary Figure S1-S4, Additional File 1). A markedly higher prevalence of cardiovascular risk factors was observed in CVD survivors (Figure 1). Figure S5 shows that CVD survivors had fewer avenues to learn about stroke.

[Figure 1]

Figures 2 and 3 show the variables independently associated with recognition of stroke symptoms and calling EMS, respectively. The CVD survivors' ability of stroke recognition and intent to call EMS did not improve with increasing number of modifiable risk factors. Moreover, out of the multiple factors, only urban-rural sites, region, education, annual income, living status, stroke awareness, number of avenues to learn about stroke, and unknown family history were statistically significant in calling EMS. Greater attempts to learn about stroke were strongly associated with both stroke recognition and prompt calling of EMS. The rate of using Internet-related avenues to gain knowledge of stroke was $9.0 \%$ (See Supplementary Table S3, Additional File 1).

[Figure 2]

[Figure 3]

\section{Discussion}

Our study shows that the knowledge of identifying stroke onset and intent to call EMS in Chinese CVD survivors were very poor and did not improve with increasing stroke risk. Fortunately, the CVD survivors were slightly more aware of the correct stroke response than the non-CVD population [13]. The rate of intent to call EMS (65.4\%)[13] was almost similar to those previously reported in China [21] (58.5\%), the United States [22] (62.9\%), and Japan [23] (81.2\%), but was markedly higher than the actual EMS usage rate in China (15.4\%-23.1\%) $[6,8,9]$. However, even in the CVD survivors with more than 3 risk factors, one-third of them would not call EMS at the onset of a recurrent stroke. 
CVD survivors are liable to experience a recurrent stroke, which is usually more disabling and costly than the first episode $[10,11,24]$. Higher prevalence of cardiovascular risk factors among CVD survivors was reported in our study, which accounted for the majority of cases of stroke onset [25]. Among the CVD survivors, multiple risk factors indicated a higher risk of recurrent stroke [25], which seems to be even higher in China due to poor control of these factors [26-29]. Unfortunately, in our study, higher stroke risk did not increase their intent to call EMS, which may delay access to reperfusion therapy $[6,9,30]$. CVD survivors probably failed to regard underlying diseases as risk factors of stroke [17, 31, 32], and did not fully understand the danger of cardiovascular risk factors, and were unaware of their situation [17], probably due to poor education on secondary stroke prevention. Therefore, comprehensive education about risk factors, recurrent stroke recognition, and calling EMS is needed to reinforce secondary prevention of stroke [31].

In contrast to results from the entire population, the odds of calling EMS among the CVD survivors depended only on a few factors, such as the region and annual income [13]. Moreover, contrary to the results from the entire group and previous studies [13,33], even highly educated CVD survivors did not perform better at recognizing stroke onset and calling EMS. Therefore, there are underlying factors other than lack of stroke education that remain unclear and require further study. This emphasizes the need to revise our previous programs to educate CVD survivors differently from the non-CVD population. In addition, doctors should optimize secondary preventive stroke-education programs to educate patients about the appropriate response to stroke onset. Moreover, the limited number of associated factors that affect the odds of calling EMS among CVD survivors imply that targeted stroke-education programs can be more efficiently designed and conducted for CVD survivors than for the non-CVD population [13].

Low education, low income, rural location, and advanced age were more common among CVD survivors, which indicated a lower equity of needs and resource allocation to EMS [34, 35] and a poor capacity to curb the stroke burden [36,37]. Additionally, our study demonstrated that having $>4$ avenues for gaining knowledge on stroke was associated with calling EMS, indicating that the CVD survivors were not sensitive to solitary education campaigns, while multiple avenues showed synergistic effects [23]. However, with the availability of point-to-point functions, Internet-related avenues appear to be more appropriate to educate specific groups, although the Internet usage rate is extremely low among Chinese CVD survivors [23, 32, 38]. Finally, since CVD survivors face extremely high prevalence of risk factors, it is better to make them recognize that good control of these modifiable risk factors can reduce stroke risk [25], and that timely and proper response to stroke onset is critical to improving the outcome [33]. Thus, we should consider these unique and unfavorable points when launching education programs for Chinese CVD survivors.

The limitations of this study include the bias due to the multistage nonrandom sampling design and selection from CNSSS, although screening sites in urban and rural areas were selected in a 1:1 ratio [18]. Moreover, even though we investigated the intent to call EMS, the respondents' actual responses to stroke remain unclear and may be overstated [22,39]. Finally, despite the stroke risk factors being extensive, we only selected seven modifiable risk factors to assess the risk for recurrent stroke. Therefore, the influence 
of the other factors remained unknown. For example, the information on intracranial arterial stenosis was not available in our study.

In conclusion, this community-based study found that stroke recognition and intent to call EMS in response to stroke-related symptoms was low among Chinese CVD survivors, and did not improve with increasing number of risk factors. Additionally, CVD survivors were characterized by low socioeconomic status and a higher prevalence of stroke risk factors. The findings indicate the need for targeted and enhanced secondary preventive stroke-education programs for CVD survivors that focus on the control of modifiable risk factors, stroke recognition, and correct response to stroke.

\section{Declarations}

\section{Acknowledgements}

We thanks Baohua Chao, Lei Cao, Lingxiao Wang, and their team for important support in the undertaking of this program.

\section{Funding}

This study was funded by the Ministry of Finance of the People's Republic of China (Issued by Finance and Social Security [2016] Document No.50, Ministry of Finance).

\section{Availability of data and materials}

The data sets in this study are available from the corresponding author on reasonable request.

\section{Authors' contribution}

BP, LW, and LYC designed the study. SL, CY, GS, and BP analyzed the data. CG finished data collection and management. SL wrote the paper. $\mathrm{LYC}, \mathrm{BP}$, and CA revised the paper.

\section{Competing interests}

Craig Anderson is employed by The George Institute China and has a National Health and Medical Research Council (NHMRC) of Australia grant. He is also a consultant for Takeda China and Amgen. The other authors declared no potential conflicts of interest.

\section{Consent for publication}


Not applicable.

\section{Ethics approval and consent to participate}

The FAST-RIGHT study was approved by the central ethics committee of Peking Union Medical College Hospital (the principal study center), and all participants provided written informed consent.

\section{Additional Material Legend}

File name: Additional file 1

File format: pdf

Tile and description of data:

Appendix1. List of the FAST-RIGHT Investigators and Coordinators

Appendix2. The structure of FAST-RIGHT questionnaire with additional 4 questions about stroke awareness

Appendix3. Definition of Risk Factors for Strokel

Appendix4. Six Regions of China in FAST-RIGHT study

Table S1. Missing data for each variable about cerebrovascular disease survivors

Table S2. Relation of recognition of stroke and correct action after stroke

Table S3. Avenues to learning about acute stroke

Figure S1. Distribution of age group between CVD survivors and non-CVD population

Figure S2. Distribution of education between CVD survivors and non-CVD population

Figure S3. Distribution of personal annual income between CVD survivors and non-CVD population

Figure S4. Distribution of BMI between CVD survivors and non-CVD population

Figure S5. Proportions of the number of avenues to gain knowledge of stroke between CVD survivors and general population

Figure S6. Data preparation and cleaning process

\section{References}


1. Feigin VL, Forouzanfar MH, Krishnamurthi R, Mensah GA, Connor M, Bennett DA, et al. Global and regional burden of stroke during 1990-2010: findings from the Global Burden of Disease Study 2010. Lancet. 2014;383 9913:245-54.

2. Liu M, Wu B, Wang WZ, Lee LM, Zhang SH, Kong LZ. Stroke in China: epidemiology, prevention, and management strategies. Lancet Neurol. 2007;6 5:456-64; doi: 10.1016/S1474-4422(07)70004-2.

3. Yang G, Wang Y, Zeng Y, Gao GF, Liang X, Zhou M, et al. 2Rapid health transition in China, 1990-2010: findings from the Global Burden of Disease Study 2010. Lancet. 2013;381 9882:1987-2015; doi: 10.1016/S0140-6736(13)61097-1.

4. de Graaf JA, van Mierlo ML, Post MWM, Achterberg WP, Kappelle LJ, Visser-Meily JMA. Long-term restrictions in participation in stroke survivors under and over 70 years of age. Disabil Rehabil. 2018;40 6:637-45; doi: 10.1080/09638288.2016.1271466.

5. Wang Y, Liao X, Zhao X, Wang DZ, Wang C, Nguyen-Huynh MN, et al. Using recombinant tissue plasminogen activator to treat acute ischemic stroke in China: analysis of the results from the Chinese National Stroke Registry (CNSR). Stroke. 2011;42 6:1658-64; doi: 10.1161/STROKEAHA.110.604249.

6. Jin H, Zhu S, Wei JW, Wang J, Liu M, Wu Y, et al. Factors associated with prehospital delays in the presentation of acute stroke in urban China. Stroke. 2012;43 2:362-70; doi:

10.1161/STROKEAHA.111.623512.

7. Saver JL, Smith EE, Fonarow GC, Reeves MJ, Zhao X, Olson DM, et al. The "golden hour" and acute brain ischemia: presenting features and lytic therapy in $>30,000$ patients arriving within 60 minutes of stroke onset. Stroke. 2010;41 7:1431-9; doi: 10.1161/STROKEAHA.110.583815.

8. Jiang B, Ru X, Sun H, Liu H, Sun D, Liu Y, et al. Pre-hospital delay and its associated factors in first-ever stroke registered in communities from three cities in China. Sci Rep. 2016;6:29795; doi:

10.1038/srep29795.

9. Yin X, Yang T, Gong Y, Zhou Y, Li W, Song X, et al. Determinants of Emergency Medical Services Utilization Among Acute Ischemic Stroke Patients in Hubei Province in China. Stroke. 2016;47 3:891-4; doi: 10.1161/STROKEAHA.115.011877.

10. Amarenco P, Lavallee PC, Monteiro Tavares L, Labreuche J, Albers GW, Abboud H, et al. Five-Year Risk of Stroke after TIA or Minor Ischemic Stroke. N Engl J Med. 2018;378 23:2182-90; doi:

10.1056/NEJMoa1802712.

11. Hankey GJ. Secondary stroke prevention. Lancet Neurol. 2014;13 2:178-94; doi: 10.1016/S14744422(13)70255-2.

12. Luengo-Fernandez R, Gray AM, Rothwell PM, Oxford Vascular S. A population-based study of hospital care costs during 5 years after transient ischemic attack and stroke. Stroke. 2012;43 12:3343-51; doi: 
13.Li S, Cui LY, Anderson C, et al. Public Awareness of Stroke and the Appropriate Responses in China: A Cross-Sectional Community-Based Study (FAST-RIGHT). Stroke. 2019;50:455-462. DOI: 10.1161/STROKEAHA.118. 023317.

14. Pan L, Yang Z, Wu Y, Yin RX, Liao Y, Wang J, et al. The prevalence, awareness, treatment and control of dyslipidemia among adults in China. Atherosclerosis. 2016;248:2-9; doi:

10.1016/j.atherosclerosis.2016.02.006.

15. Lu J, Lu Y, Wang X, Li X, Linderman GC, Wu C, et al. Prevalence, awareness, treatment, and control of hypertension in China: data from 1.7 million adults in a population-based screening study (China PEACE Million Persons Project). Lancet. 2017;390 10112:2549-58; doi: 10.1016/S0140-6736(17)32478-9.

16. Qin Y, Wang R, Ma X, Zhao Y, Lu J, Wu C, et al. Prevalence, Awareness, Treatment and Control of Diabetes Mellitus-A Population Based Study in Shanghai, China. Int J Environ Res Public Health. 2016;13 5; doi: 10.3390/ijerph13050512.

17. Slark J, Bentley P, Majeed A, Sharma P. Awareness of stroke symptomatology and cardiovascular risk factors amongst stroke survivors. J Stroke Cerebrovasc Dis. 2012;21 5:358-62; doi:

10.1016/j.jstrokecerebrovasdis.2010.09.010.

18. Longde $\mathrm{W}$, Ling $\mathrm{Y}$, Yang $\mathrm{H}, \mathrm{Yi} Z$ Z, Yongjun $\mathrm{W}$, Xunming J, et al. Fixed-dose combination treatment after stroke for secondary prevention in China: a national community-based study. Stroke. 2015;46 5:1295-300; doi: 10.1161/STROKEAHA.114.007384.

19.National Center for Stroke Control and Prevention, National Health Commission. The China National Stroke Screening Survey Guidelines [online, in Chinese]. http://cnstroke.com/WebManage/InterveneProject/Index. (2016, accessed 28 May 2019).

20. Harbison J, Hossain O, Jenkinson D, Davis J, Louw SJ, Ford GA. Diagnostic accuracy of stroke referrals from primary care, emergency room physicians, and ambulance staff using the face arm speech test. Stroke. 2003;34 1:71-6.

21. Yang J, Zheng M, Cheng S, Ou S, Zhang J, Wang N, et al. Knowledge of stroke symptoms and treatment among community residents in Western Urban China. J Stroke Cerebrovasc Dis. 2014;23 5:1216-24; doi: 10.1016/j.jstrokecerebrovasdis.2013.10.019.

22. Fussman C, Rafferty AP, Lyon-Callo S, Morgenstern LB, Reeves MJ. Lack of association between stroke symptom knowledge and intent to call 911: a population-based survey. Stroke. 2010;41 7:1501-7; doi: 10.1161/STROKEAHA.110.578195.

23. Miyamatsu N, Okamura T, Nakayama H, Toyoda K, Suzuki K, Toyota A, et al. Public awareness of early symptoms of stroke and information sources about stroke among the general Japanese population: 
the Acquisition of Stroke Knowledge Study. Cerebrovasc Dis. 2013;35 3:241-9; doi: 10.1159/000347066.

24. Wang YL, Pan YS, Zhao XQ, Wang D, Johnston SC, Liu LP, et al. Recurrent stroke was associated with poor quality of life in patients with transient ischemic attack or minor stroke: finding from the CHANCE trial. CNS Neurosci Ther. 2014;20 12:1029-35; doi: 10.1111/cns.12329.

25. O'Donnell MJ, Chin SL, Rangarajan S, Xavier D, Liu L, Zhang H, et al. Global and regional effects of potentially modifiable risk factors associated with acute stroke in 32 countries (INTERSTROKE): a casecontrol study. Lancet. 2016;388 10046:761-75; doi: 10.1016/S0140-6736(16)30506-2.

26. Wei Y, Xu J, Wu H, Zhou G, Chen S, Wang C, et al. Survey of Antithrombotic Treatment in Rural Patients (>60 years) with Atrial Fibrillation in East China. Sci Rep. 2018;8 1:6830; doi: 10.1038/s41598018-24878-y.

27. Yang F, Qian D, Chen J, Hu D, Hou M, Chen S, et al. Prevalence, awareness, treatment and control of diabetes mellitus in rural China: results from Shandong Province. Diabet Med. 2016;33 4:454-8; doi: $10.1111 / \mathrm{dme} .12842$.

28. Zhao Y, Yan H, Marshall RJ, Dang S, Yang R, Li Q, et al. Trends in population blood pressure and prevalence, awareness, treatment, and control of hypertension among middle-aged and older adults in a rural area of Northwest China from 1982 to 2010. PLoS One. 2013;8 4:e61779; doi: 10.1371/journal.pone.0061779.

29. Chen Z, Peto R, Zhou M, lona A, Smith M, Yang L, et al. Contrasting male and female trends in tobacco-attributed mortality in China: evidence from successive nationwide prospective cohort studies. Lancet. 2015;386 10002:1447-56; doi: 10.1016/S0140-6736(15)00340-2.

30. Fonarow GC, Smith EE, Saver JL, Reeves MJ, Bhatt DL, Grau-Sepulveda MV, et al. Timeliness of tissuetype plasminogen activator therapy in acute ischemic stroke: patient characteristics, hospital factors, and outcomes associated with door-to-needle times within 60 minutes. Circulation. 2011;123 7:750-8; doi: 10.1161/CIRCULATIONAHA.110.974675.

31. Das K, Mondal GP, Dutta AK, Mukherjee B, Mukherjee BB. Awareness of warning symptoms and risk factors of stroke in the general population and in survivors stroke. J Clin Neurosci. 2007;14 1:12-6; doi: 10.1016/j.jocn.2005.12.049.

32. Metias MM, Eisenberg N, Clemente MD, Wooster EM, Dueck AD, Wooster DL, et al. Public health campaigns and their effect on stroke knowledge in a high-risk urban population: A five-year study. Vascular. 2017;25 5:497-503; doi: 10.1177/1708538117691879.

33. Fassbender K, Balucani C, Walter S, Levine SR, Haass A, Grotta J. Streamlining of prehospital stroke management: the golden hour. Lancet Neurol. 2013;12 6:585-96; doi: 10.1016/S1474-4422(13)70100-5. 
34. Liu Y, Jiang Y, Tang S, Qiu J, Zhong X, Wang Y. Analysis of the equity of emergency medical services: a cross-sectional survey in Chongqing city. Int J Equity Health. 2015;14:150; doi: 10.1186/s12939-0150282-8.

35. Yan K, Jiang Y, Qiu J, Zhong X, Wang Y, Deng J, et al. The equity of China's emergency medical services from 2010-2014. Int J Equity Health. 2017;16 1:10; doi: 10.1186/s12939-016-0507-5.

36. Pan Y, Song T, Chen R, Li H, Zhao X, Liu L, et al. Socioeconomic deprivation and mortality in people after ischemic stroke: The China National Stroke Registry. Int J Stroke. 2016;11 5:557-64; doi:

$10.1177 / 1747493016641121$.

37. Cha JK, Lim JH, Kim DH, Nah HW, Park HS, Choi JH, et al. Prognostic factors for long-term poor outcomes after acute ischemic stroke in very old age ( $>80$ years) patients: Total cholesterol level might differently influence long-term outcomes after acute ischemic stroke at ages above 80 years. Geriatr Gerontol Int. 2015;15 11:1227-33; doi: 10.1111/ggi.12419.

38. Kim YS, Park SS, Bae HJ, Heo JH, Kwon SU, Lee BC, et al. Public awareness of stroke in Korea: a population-based national survey. Stroke. 2012;43 4:1146-9; doi: 10.1161/STROKEAHA.111.638460.

39. Teuschl Y, Brainin M. Stroke education: discrepancies among factors influencing prehospital delay and stroke knowledge. Int J Stroke. 2010;5 3:187-208; doi: 10.1111/j.1747-4949.2010.00428.x.

\section{Tables}

Table1. SRR and CAR by demographic, socio-economic, and stroke risk variables in CVD survivors 


\begin{tabular}{|c|c|c|c|c|}
\hline & SRR & & CAR & \\
\hline & $\mathrm{n} / \mathrm{N}(\%, 95 \% \mathrm{CI})$ & $p$ value & $\mathrm{n} / \mathrm{N}(\%, 95 \% \mathrm{CI})$ & $p$ value \\
\hline Age (years) & & $<0.0001$ & & 0.0038 \\
\hline $40-49$ & 260/301 (86.4\%, & & 200/301 (66.5\%, & \\
\hline & $82.5-90.3)$ & & 61.1-71.8) & \\
\hline $50-59$ & $921 / 1039$ (88.6\%, & & 723/1039 (69.59\%, & \\
\hline & 86.7-90.6) & & 66.79-72.38) & \\
\hline $60-69$ & 2070/2376 (87.1\%, & & $1560 / 2376$ (65.66\%, & \\
\hline & 85.8-88.5) & & 63.75-67.57) & \\
\hline $70-79$ & 1604/1943 (82.6\%, & & $1249 / 1943$ (64.28\%, & \\
\hline & 80.9-84.2) & & $62.15-66.41)$ & \\
\hline 80-99 & 493/631 (78.1\%, & & $383 / 631(60.70 \%$, & \\
\hline & 74.9-81.4) & & $56.89-64.51)$ & \\
\hline Sex & & 0.8873 & & 0.6738 \\
\hline Male & $2496 / 2938$ (85.0\%, & & 1930/2938 (65.7\%, & \\
\hline & 83.7-86.3) & & $64.0-67.4)$ & \\
\hline Female & 2852/3352 (85.1\%, & & 2185/3352 (65.2\%, & \\
\hline & 83.9-86.3) & & 63.6-66.8) & \\
\hline Site & & 0.0018 & & $<0.0001$ \\
\hline Urban & 2537/2932 (86.5\%, & & 2195/2932 (74.9\%, & \\
\hline & 85.3-87.8) & & 73.3-76.4) & \\
\hline Rural & 2811/3358 (83.7\%, & & 1920/3358 (57.2\%, & \\
\hline & 82.5-85.0) & & 55.5-58.9) & \\
\hline Regions & & $<0.0001$ & & $<0.0001$ \\
\hline North + Northeast & $684 / 718$ (95.3\%, & & $490 / 718(68.3 \%$, & \\
\hline & 93.7-96.8) & & 64.8-71.7) & \\
\hline East & $1291 / 1510(85.5 \%$ & & $848 / 1510(56.2 \%$, & \\
\hline & 83.7-87.3) & & $53.7-58.7)$ & \\
\hline Central & $2306 / 2726$ (84.6\%, & & 1933/2726 (70.9\%, & \\
\hline & 83.2-86.0) & & 69.2-72.6) & \\
\hline
\end{tabular}


South

Southwest

Northwest

250/322 (77.6\%,

73.1-82.2)

Education

$\leq$ Primary

2624/3247 (80.8\%,

79.5-82.2)

Middle/High school 2442/2733 (89.4\%,

88.2-90.5)

$\geq$ College

282/310 (91.0\%,

87.8-94.2)
232/392 (59.2\%,

54.3-64.1)

445/622 (71.5\%, 68.0-75.1)

167/322 (51.9\%,

46.4-57.3)

$<0.0001$

1860/3247 (57.3\%,

55.6-59.0)

2015/2733 (73.7\%,

72.1-75.4)

240/310 (77.4\%,

72.8-82.1)
1394/2479 (56.2\%,

54.3-58.2)

1365/2005 (68.1\%,

66.0-70.1)

1356/1803 (75.2\%,

73.2-77.2)

0.1933

$4982 / 5849$ (85.2\%,

84.27-86.1)

363/438 (82.9\%,

79.4-86.4)

$<0.0001$

47/57 (82.5\%, 72.58-

92.33)

1122/1228 (91.4\%,

$<0.0001$

$<0.0001$

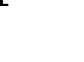

Living Status ${ }^{a}$

With family

Children number

0 
89.8-92.9)

2-3

$\geq 4$

3309/3876 (85.4\%,

84.3-86.5)

865/1124 (77.0\%,

74.5-79.4)

Awareness of stroke

No

Yes

Avenues $^{\mathrm{b}}$

1

2-3

4-6

2455/3103 (79.1\%,

77.7-80.6)

2646/2936 (90.1\%,

89.0-91.2)

247/251 (98.4\%,

96.9-99.96)
73.4-78.3)

2494/3876 (64.3\%,

62.8-65.9)

643/1124 (57.2\%,

54.3-60.1)

..

$<0.0001$

482/942 (51.2\%,

48.0-54.4)

3633/5348 (67.9\%, 66.7-69.2)

$<0.0001$

1987/3103 (64.0\%,

62.4-65.7)

1919/2936 (65.4\%,

63.6-67.1)

209/251 (83.3\%,

78.7-87.9)

$<0.0001$

Number of risk

0.4279

factors $^{\mathrm{C}}$

0

321/379 (84.7\%,

250/379 (66.0\%,

81.1-88.3)

61.2-70.7)

$1-2$

2346/2780 (84.4\%,

1744/2780 (62.7\%,

83.0-85.7)

60.1-64.5)

3-7

2673/3123 (85.6\%,

84.4-86.8)

2117/3123 (67.8\%,

66.2-69.4)

SRR: Stroke recognition rate; CAR: correct action rate.

$\mathrm{N}$ : total in every variable; $\mathrm{n}$ : number for recognizing stroke/correct action to stroke. 
a With family includes living with spouse/children; With others includes being single, living in a nursing home, and with other people

$\mathrm{b}$ The number of avenues taken to learn about acute stroke

C The number of modifiable risk factors, its range was 0 to 7 .

\section{Figures}
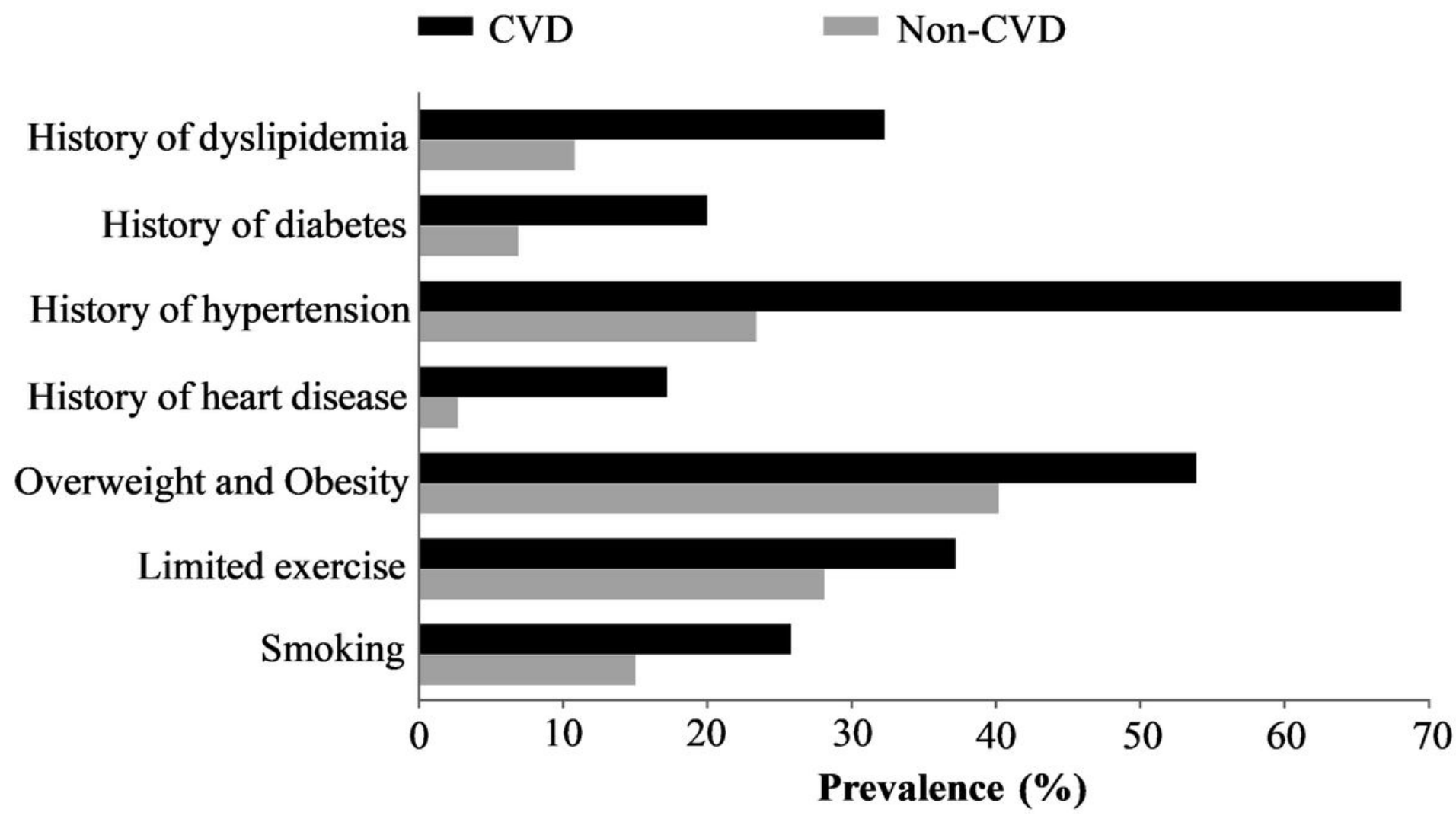

Figure 1

Prevalence of cardiovascular risk factors between CVD survivors and non-CVD population CVD: CVD survivors, with history of cerebral vascular disease Non-CVD: Adults without history of cerebral vascular disease 


\section{OR $(95 \% \mathrm{CI})$}

Age, y (Ref: 40-49)

50-59

$1.24(0.84-1.85)$

60-69

$1.25(0.86-1.80)$

70-79

$1.04(0.71-1.50)$

80-99

$0.79(0.52-1.19)$

Female (Ref: Male)

$1.14(0.98-1.32)$

Rural (Ref: Urban)

$1.42(1.19-1.69)$

Regions (Ref: North + Northeast)

East

Central

South

Southwest

Northwest

Education (Ref: $\leq$ Primary)

Middle/High school

$\geq$ College

$1.32(0.86-2.05)$

Personal Annual Income (US \$) (Ref: < 731)

731-2923

$1.03(0.87-1.23)$

$>2923$

$2.19(1.72-2.80)$

$0.92(0.69-1.22)$

Children number (Ref: 0)

1

$1.38(0.64-3.00)$

$1.05(0.49-2.22)$

$0.78(0.36-1.67)$

$\geq 4$

Avenues $^{\text {b }}$ (Ref: 1)

$2-3$

$2.11(1.81-2.47)$

$15.26(5.57-41.82)$

4-6

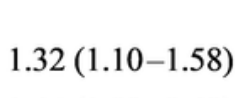

$1.64(1.03-2.62)$

Unknown

Number of risk factors ${ }^{\mathrm{c}}($ Ref: 0$)$

$1-2$

$0.99(0.72-1.34)$

3-7

$0.97(0.71-1.33)$

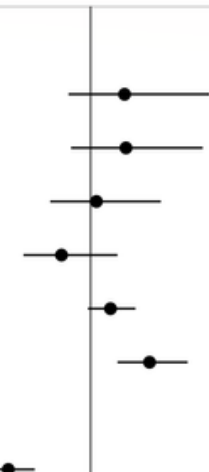


OR (95\%CI)

Age, y (Ref: 40-49)

$50-59$

60-69

70-79

80-99

Female (Ref: Male)

Rural (Ref: Urban)

Regions (Ref: North + Northeast)

East

Central

South

Southwest

Northwest

Education (Ref: $\leq$ Primary)

Middle/High school

$\geq$ College

Personal Annual Income (US \$) (Ref: < 731)

$731-2923$

$>2923$

Living with others ${ }^{\text {a }}$ (Ref: With family)

Children number (Ref: 0)

1

$2-3$

$\geq 4$

Awareness of stroke

Avenues $^{\text {b }}$ (Ref: 1)

$2-3$

4-6

Family history of stroke (Ref: No)

Yes

Unknown

Number of risk factors ${ }^{\mathrm{c}}$ (Ref: 0$)$

$1-2$

3-7
$1.03(0.77-1.37)$

$0.92(0.70-1.21)$

$0.95(0.72-1.26)$

$0.82(0.59-1.12)$

$1.08(0.97-1.21)$

$0.56(0.49-0.64)$

$0.69(0.56-0.85)$

$1.36(1.12-1.65)$

0.75 (0.57-0.99)

1.61 (1.25-2.08)

$0.42(0.31-0.57)$

$1.48(1.30-1.68)$

$1.18(0.87-1.60)$

1.39 (1.21-1.59)

1.42 (1.19-1.69)

1.59 (1.25-2.00)

$0.70(0.36-1.39)$

0.59 (0.31-1.16)

$0.58(0.30-1.14)$

1.80 (1.55-2.10)

$0.98(0.87-1.10)$

2.43 (1.68-3.52)

0.95 (0.83-1.09)

$0.73(0.54-0.998)$

$0.83(0.65-1.05)$

0.97 (0.76-1.24)

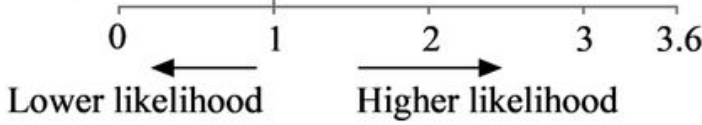

to call EMS to call EMS

\section{Figure 3}

Logistic regression models of factors associated with calling EMS after stroke a With family includes living with spouse/children; With others includes being single, living in a nursing home, and with other people $b$ Avenues taken to learn about acute stroke $c$ The number of modifiable risk factors, its range was 0 to 7 . 


\section{Supplementary Files}

This is a list of supplementary files associated with this preprint. Click to download.

- supplement1.pdf 